\title{
Yarn Motion in Interlacers with Various Cross-Sectional Shapes of Yarn Duct
}

\author{
QIU Hua, IEMOTO Yoshiyuki*, TANOUE Shuichi \\ Graduate School of Engineering, University of Fukui, 3-9-1 Bunkyo, Fukui 910-8507, Japan
}

Received 13 January 2007; accepted for publication 14 March 2007

\begin{abstract}
An investigation of yarn motion in yarn ducts with various cross-sectional shapes and its effects on the characteristics of interlaced yarn is reported. Seven interlacers, which are different in the cross-sectional shape of yarn duct, have the same cross-sectional area. Yarn motion in the yarn duct was observed with 16 sets of optical fibers and photo-sensors. The characteristics of the interlaced yarn were evaluated by both the number and the strength of tangles. The interlacer with a round yarn duct is capable of producing an interlaced yarn with larger number of tangles. This is because it can make yarn run frequently across an air jet issuing from an air jet nozzle. On the other hand, the interlacer with a cornered yarn duct makes yarn subjected to the strong action of a compressed air for a longer time, thus it results in higher strength of tangles.
\end{abstract}

Key Words: Interlacer, Shapes of yarn duct, Interlaced yarn, Observation of yarn motion

\section{Introduction}

Interlacing is an important method to improve cohesive force between filaments of chemical fiber. During interlacing, a loose bundle of filaments is subjected to the action of compressed air issuing from the direction perpendicular to the yarn axis. As a result, the opening parts and the tangling parts are formed intermittently in an interlaced yarn [1]. The cost of interlacing is seven to nine times cheaper than that of sizing and twisting [2]. Since the first patent about interlacing process was published in 1961 [3], many researches have been carried out [4-9].

We have carried out a research about the effect of seven cross-sectional shapes of yarn duct on the production performance of interlacers [10]. Production performance of the interlacers was evaluated by both the number and the strength of tangles in the interlaced yarn. The cross-sectional shapes and sizes of the seven yarn ducts are shown in Fig. 1. These seven yarn ducts have the same cross-sectional area. In Fig. 1, notation $\mathrm{C} 1$ represents the interlacer with a circular cross-sectional yarn duct. E1, E2 and E3 are interlacers with an elliptical yarn duct. S1 has a square yarn duct; T1 and T2 have a triangular one. These seven interlacers can be categorized into two classes: yarn duct with round shape, E1, E2, C1 and E3; yarn duct with cornered shape, S1, T1 and T2. Owing to the difference of

cross-sectional shapes of yarn duct, yarn motion is different in each yarn duct, thus the production performance of each interlacer is largely affected. In the previous researches [2, 11-13], yarn posture and motion in the circular yarn duct

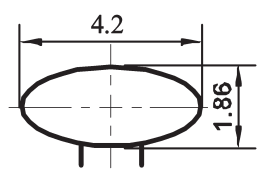

E1

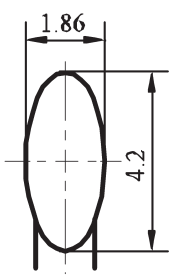

E3

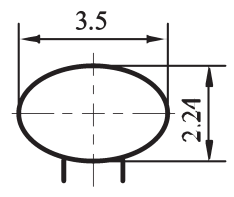

E2

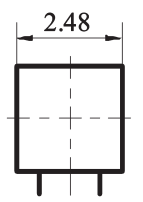

S1

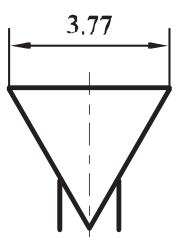

T2

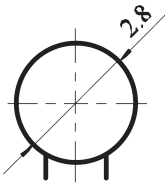

C1

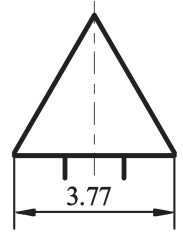

T1

* Corresponding author: E-mail: iemoto@matse.fukui-u.ac.jp, Tel: +81-776-27-8625, Fax: +81-776-27-8767 
were studied.

In this study, we observed the yarn motion in the yarn ducts with seven cross-sectional shapes. The yarn motion was observed with 16 sets of optical fibers and photosensors. Furthermore, effects of processing parameters on the yarn motion in the yarn duct were investigated. On the basis of the characteristics of the interlaced yarn, we got the interlacers with better performance, and the relationship between the yarn motion and the cross-sectional shape of the yarn duct was also elucidated.

\section{Experiments}

A raw yarn made of polyester multi-filament, 16.7 tex/48 filaments was used as a tested material.

An apparatus for interlacing process is the same as the previous study [10], so it is omitted here. Dimensions of the interlacers and coordinate system of the yarn duct crosssection are shown in Fig. 2 (taking the interlacer E2 as an example). As shown in Fig. 2(a), the interlacer is composed of an air jet nozzle (1), a yarn duct (2) and two yarn guides (3). Diameter of air jet nozzle is $1.4 \mathrm{~mm}$, length of yarn duct is $14.7 \mathrm{~mm}$ and distance between two yarn guides is $25.4 \mathrm{~mm}$. The air jet nozzle is positioned at the bottom of yarn duct. The coordinates $x$ and $y$ are used to represent yarn positions. Figure 2(b) shows an interlacer for measurement of yarn motion. It is composed of two parts (1), (2). A yarn ran from left to right, and the slits (3) were used for inserting optical fibers of $0.25 \mathrm{~mm}$ in diameter. The best cross-section of yarn duct for observing yarn motion is the OO' section including the axis of the air jet nozzle as shown in Fig. 2(a) because yarn motion at the OO' section characterizes interlacing process. However, the air jet nozzle blocks the optical fiber insertion. Thus, optical fibers were set $1.45 \mathrm{~mm}$ apart from the axis of the air jet nozzle. For each interlacer, 16 pairs of optical fibers were used to measure yarn motion. The 16 light-receiving optical fibers were put face to face with the 16 light-serving optical fibers. Figure 3 shows the arrangement of optical fibers in the interlacer E2. The distributions of optical fibers in the $x$ and $y$ directions depended on the height and width of the yarn duct.

Since the cross-sectional area of tested yarn used in this experiment is about $0.17 \mathrm{~mm}^{2}$, the yarn diameter is about $0.47 \mathrm{~mm}$ when the cross-sectional shape of the yarn is assumed to be circular. Hence, the yarn staying between a pair of optical fibers can completely block the light radiated from the light-serving optical fiber because the optical fiber diameter is $0.25 \mathrm{~mm}$ [13]. In the case of the interlacer E2, optical fibers $\mathrm{O}_{1}, \mathrm{O}_{2}, \cdots, \mathrm{O}_{10}$ detected yarn position in the $x$ direction and $\mathrm{O}_{11}, \mathrm{O}_{12}, \cdots, \mathrm{O}_{16}$ detected it in the $y$ direction. In measurement circuit, a photodiode converted the intensity

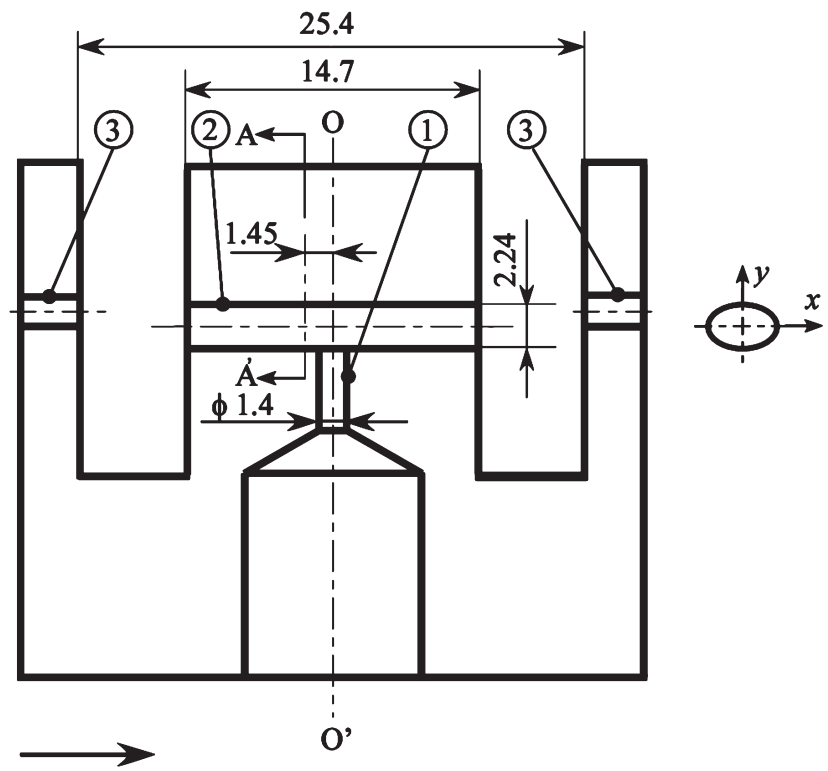

Yarn running direction

(1) Air jet nozzle (2) Yarn duct (3) Yarn guide

(a) Dimensions of the interlacers and coordinate system of the yarn duct cross-section (unit: $\mathrm{mm}$ )

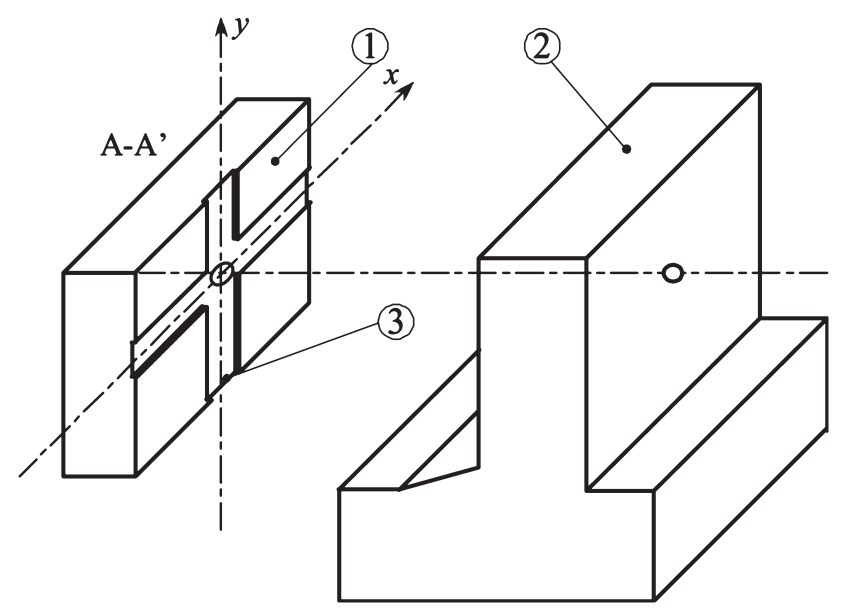

(1) A part of yarn duct with slits for inserting optical fibers

(2) A part of yarn duct with air jet nozzle

(3) Slit

(b) Interlacer for measurement of yarn motion

Fig. 2 Interlacer.

of light into the voltage. The signals received by the 16 optical fibers were simultaneously input to a computer and the yarn positions at the measurement cross-section were recorded. When the filaments constituting a yarn were separated in the yarn duct under the action of compressed air and more than one optical fiber detected yarn existence, we decided the yarn position from the arithmetic mean of position coordinates of the optical fibers. Sampling frequency was $50 \mathrm{kHz}$ and the amount of data collected in 


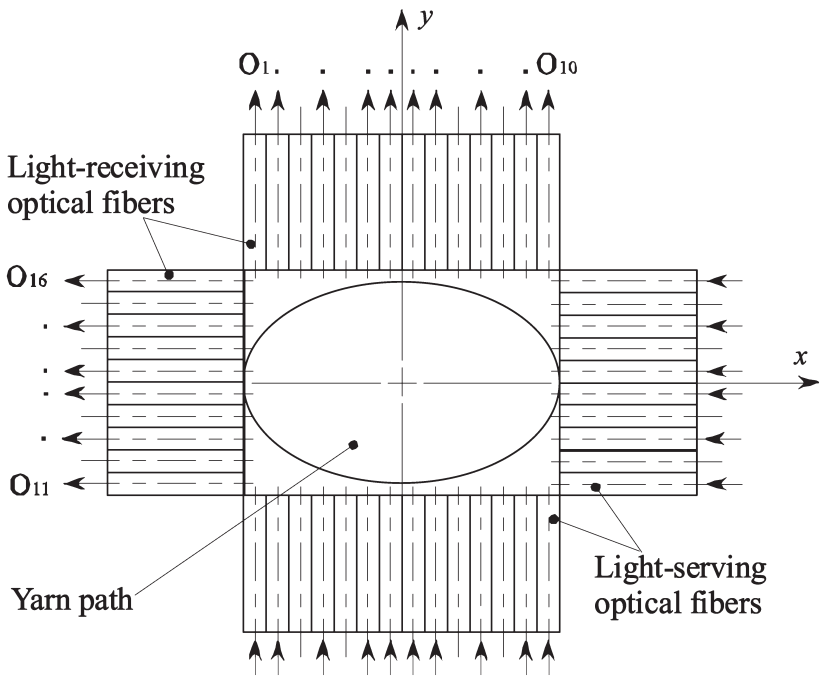

Fig. 3 Arrangement of optical fibers in the interlacer E2.

each test was 32000 , i.e. the time interval between two data in each measurement is $20 \mu \mathrm{s}$ and the time for each test is $640 \mathrm{~ms}$.

When the compressed air was not issued, the yarn was positioned at the half height of the cross-section of yarn duct on the symmetric plane including the axes of the yarn duct and the air jet nozzle. During experiment, initial yarn tension was fixed at $4.41 \mathrm{mN} / \mathrm{tex}$. To compare yarn motions in the seven yarn ducts, interlaced yarns were produced at the supplied air pressure of $0.3 \mathrm{MPa}$ (gauge pressure), the yarn speed of $200 \mathrm{~m} / \mathrm{min}$ and the feed ratio of $2 \%$ because we could produce interlaced yarns with high quality at this combination of experimental conditions [10]. Furthermore, yarn motions in the interlacers E2 and T2 were observed on some other experimental conditions to investigate effects of the processing parameters on the yarn motion in the yarn duct.

In order to evaluate the performance of the interlacers, the number of tangles $N$ was defined as the number of tangling parts in an interlaced yarn per meter. The strength of tangles $S$ was defined as $N_{r} / N$, where $N_{r}$ is the residual tangling number in the interlaced yarn per meter after the action of a load of $132 \mathrm{mN} /$ tex for $3 \mathrm{~min}$ [14]. When $N$ is small, even if $S$ is much higher, the quality of the interlaced yarn is bad, i.e. production performance of the interlacer is bad. Thus the value of $N$ is essential in evaluating the quality of an interlaced yarn, larger $N$ indicates the fine tangling degree of filaments.

\section{Results and discussion}

Figure 4 shows the number of tangles $N$ and the strength of tangles $S$ of the interlaced yarn produced by the seven interlacers at the supplied air pressure $p=0.3 \mathrm{MPa}$, the yarn

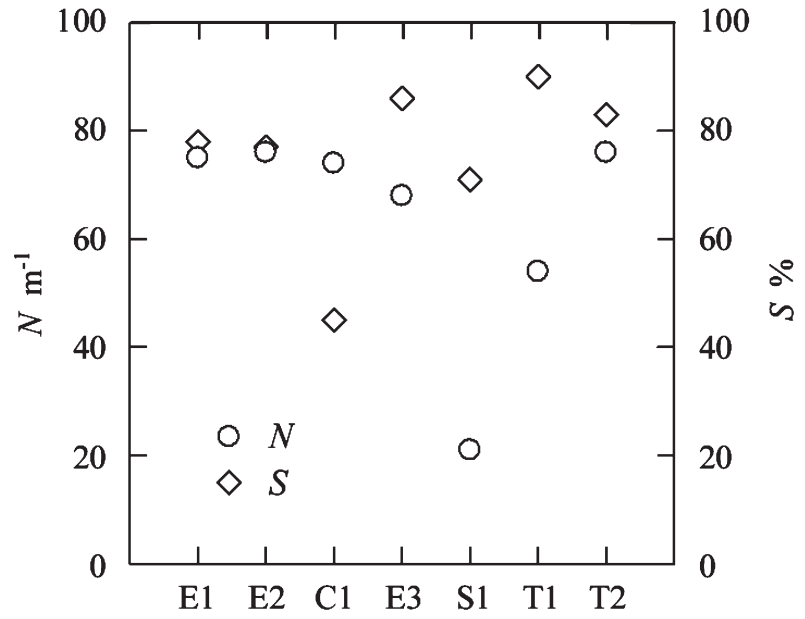

Cross-sectional shape of yarn duct

Fig. 4 The number of tangles $N$ and the strength of tangles $S$ of the interlaced yarn produced by seven interlacers at the supplied air pressure of $0.3 \mathrm{MPa}$, the yarn speed of 200 $\mathrm{m} / \mathrm{min}$ and the feed ratio of $2 \%$ [10].

Table 1 Comparison of the number of tangles $N$ and the strength of tangles $S$ between the interlacers E2 and T2 at the feed ratio of $2 \%$ [10].

\begin{tabular}{|c|c|c|c|c|}
\hline Interlacer & $p \mathrm{MPa}$ & $v \mathrm{~m} / \mathrm{min}$ & $N \mathrm{~m}^{-1}$ & $S \%$ \\
\hline \multirow{3}{*}{ E2 } & 0.1 & 200 & 68 & 75 \\
\cline { 2 - 5 } & 0.5 & 200 & 75 & 73 \\
\cline { 2 - 5 } & 0.3 & 600 & 67 & 87 \\
\hline \multirow{3}{*}{$\mathrm{T} 2$} & 0.1 & 200 & 68 & 81 \\
\cline { 2 - 5 } & 0.5 & 200 & 71 & 80 \\
\cline { 2 - 5 } & 0.3 & 600 & 63 & 88 \\
\hline
\end{tabular}

speed $v=200 \mathrm{~m} / \mathrm{min}$ and the feed ratio $F=2 \%$. Table 1 shows the comparison of $N$ and $S$ between the interlacers E2 and $\mathrm{T} 2$ at $F=2 \%$. These data were reproduced from the previous paper [10].

\section{1 Yarn motion in the yarn ducts with a round shape}

The interlacers E1, E2, C1 and E3 are classed as the interlacer with a round yarn duct. The interlacer E1 has the lowest yarn duct height and the interlacer E3 has the highest. Among these four interlacers, from Fig. 4, the interlacer E2 can produce an interlaced yarn with the largest $N$; the interlacer E3 can produce an interlaced yarn with the highest $S$ and the smallest $N$; and the interlaced yarn produced with the interlacer $\mathrm{C} 1$ has the lowest $S$. To compare yarn motions in the interlacers E1, E2, C1 and E3, observations were carried out at $p=0.3 \mathrm{MPa}, v=200 \mathrm{~m} / \mathrm{min}$ and $F=2 \%$.

Figure 5 shows the yarn motion and positions in the interlacer E1. Figures 5(a) and (b) show yarn positions in the $x$ and $y$ directions as a function of time $t$ and at the measurement 


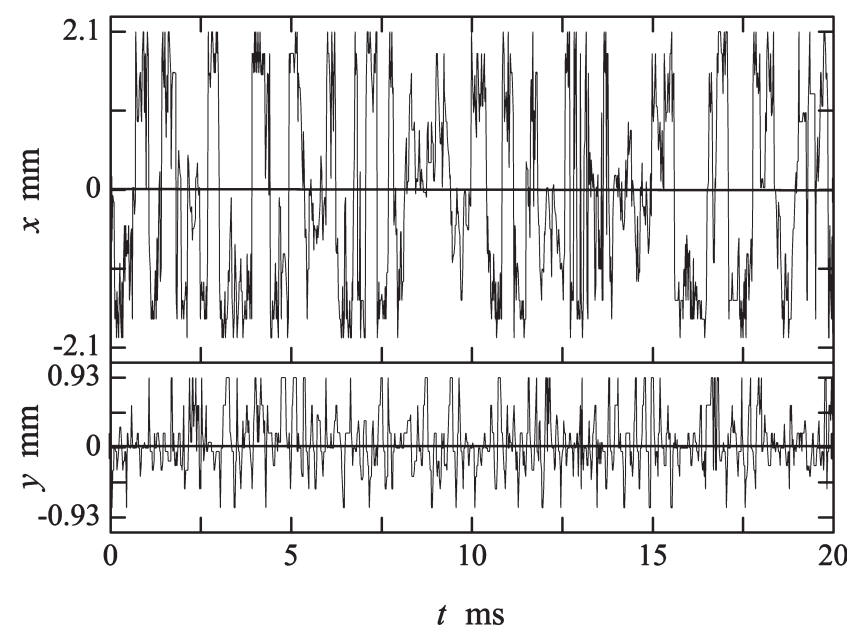

(a) Yarn positions in the $x$ and $y$ directions as a function of time $t$

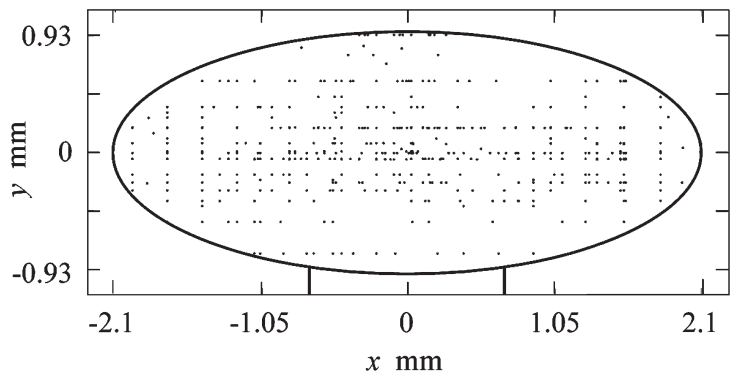

(b) Yarn positions for $20 \mathrm{~ms}$

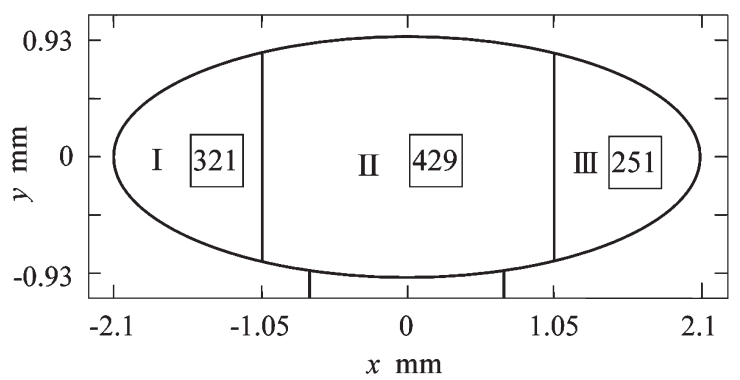

(c) Amount of yarn positions appearing within the three regions for $20 \mathrm{~ms}$

Fig. 5 Yarn motion and positions in the interlacer E1 at the supplied air pressure of $0.3 \mathrm{MPa}$, the yarn speed of 200 $\mathrm{m} / \mathrm{min}$ and the feed ratio of $2 \%$.

cross-section for $20 \mathrm{~ms}$, respectively. In Fig. 5(b) some yarn positions overlap each other. Figure 5(c) shows the amount of yarn positions in three regions, I: $x \leq-1.05 \mathrm{~mm}, \mathrm{II}:-1.05 \mathrm{~mm}$ $<x<1.05 \mathrm{~mm}$, III : $x \geq 1.05 \mathrm{~mm}$. The amount of yarn positions in each region is indicated with the Arabic number in a small square. Among total 1001 yarn positions, $572(=321+251)$ yarn positions appear in the regions $|x| \geq 1.05 \mathrm{~mm}$. Although yarn stays in the two extreme side regions of the yarn duct for a relatively long time, yarn moves in a wide space from one extreme side region to the other and frequently runs across the air jet.

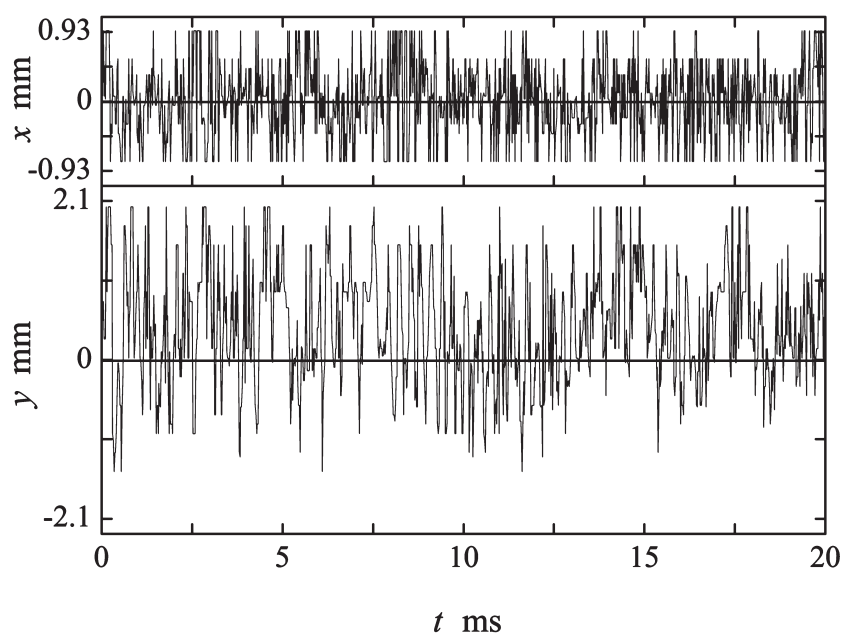

(a) Yarn positions in the $x$ and $y$ directions as a function of time $t$
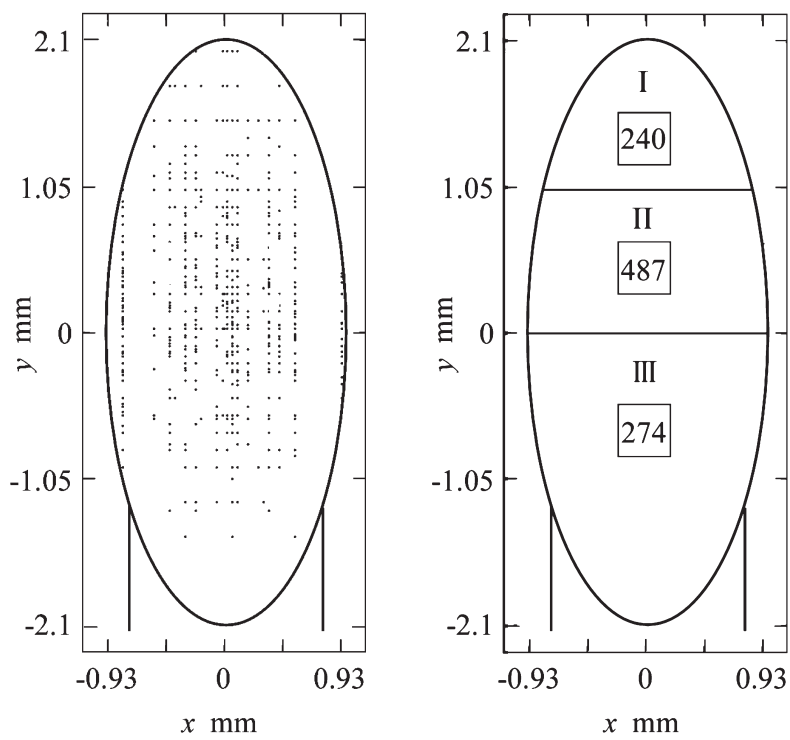

(b) Yarn positions for $20 \mathrm{~ms}$

(c) Amount of yarn positions appearing within the three regions for $20 \mathrm{~ms}$

Fig. 6 Yarn motion and positions in the interlacer E3 at the supplied air pressure of $0.3 \mathrm{MPa}$, the yarn speed of 200 $\mathrm{m} / \mathrm{min}$ and the feed ratio of $2 \%$.

Figure 6 shows the yarn motion and positions in the interlacer E3. Figures 6(a) and (b) show yarn positions in the $x$ and $y$ directions as a function of time $t$ and at the measurement cross-section for $20 \mathrm{~ms}$, respectively. Figure 6(c) shows the amount of yarn positions in three regions, I: $y \geq 1.05 \mathrm{~mm}$, II : $0 \mathrm{~mm} \leq y<1.05 \mathrm{~mm}$, III : $y<0 \mathrm{~mm}$. From Figs. 6(b) and (c), yarns of $73 \%$ are positioned in the region $y \geq 0 \mathrm{~mm}$. Yarn mainly stays in the upper half region of the yarn duct, furthermore yarns of $24 \%$ are positioned in the upper quarter region $y \geq 1.05 \mathrm{~mm}$. Since the diameter $(1.4 \mathrm{~mm})$ of air jet nozzle is close to the width $(1.86 \mathrm{~mm})$ of yarn duct, yarn is always subjected to the direct action of the compressed air issuing from the air jet nozzle. The intensity of the airflow is so strong that the yarn seldom runs to the 
lower region of the yarn duct as shown in Fig. 6(a). Yarn is subjected to the compressed air in a narrow space, thus the yarn cannot move smoothly and $S$ is the highest.

Figures 7(a) and (b) show the yarn motion and trace from $t=0.18 \mathrm{~ms}$ to $0.60 \mathrm{~ms}$ in the interlacer E2, respectively. In Fig. 7(b), the center of the small circles indicates the yarn position and the number in each small circle gives the order of the yarn positions, and also the increment by 1 means time progress of $20 \mu \mathrm{s}$. Figures 8(a) and (b) show the yarn motion and trace from $t=10.64 \mathrm{~ms}$ to $11.06 \mathrm{~ms}$ in the interlacer $\mathrm{C} 1$, respectively.

From Figs. 7(a) and 8(a), owing to the strong intensity of the airflow near the exit of air jet nozzle, yarn cannot run to the bottom of the yarn duct in the interlacers E2 and C1. However, yarn can run between the top of the yarn duct and the vicinity of the bottom of the yarn duct, which means that the yarn can be interlaced in a bigger space. In the interlacer $\mathrm{C} 1$, yarn can run across the air jet more frequently and

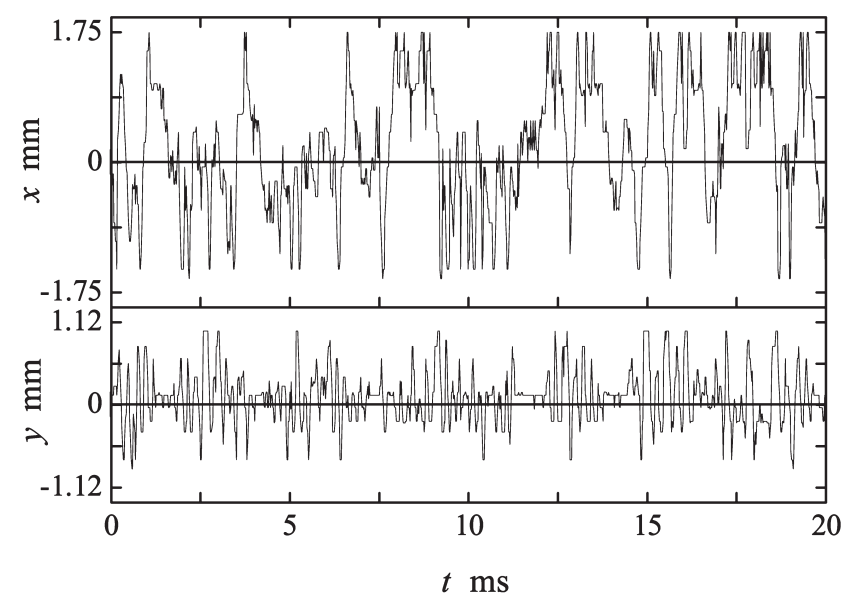

(a) Yarn positions in the $x$ and $y$ directions as a function of time $t$

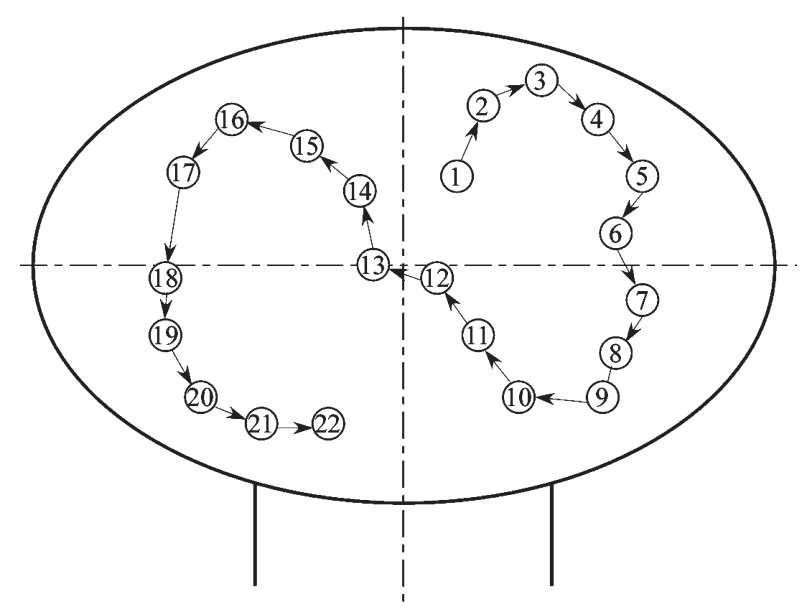

(b) Yarn trace from $0.18 \mathrm{~ms}$ to $0.60 \mathrm{~ms}$

Fig. 7 Yarn motion and trace in the interlacer E2 at the supplied air pressure of $0.3 \mathrm{MPa}$, the yarn speed of $200 \mathrm{~m} / \mathrm{min}$ and the feed ratio of $2 \%$.

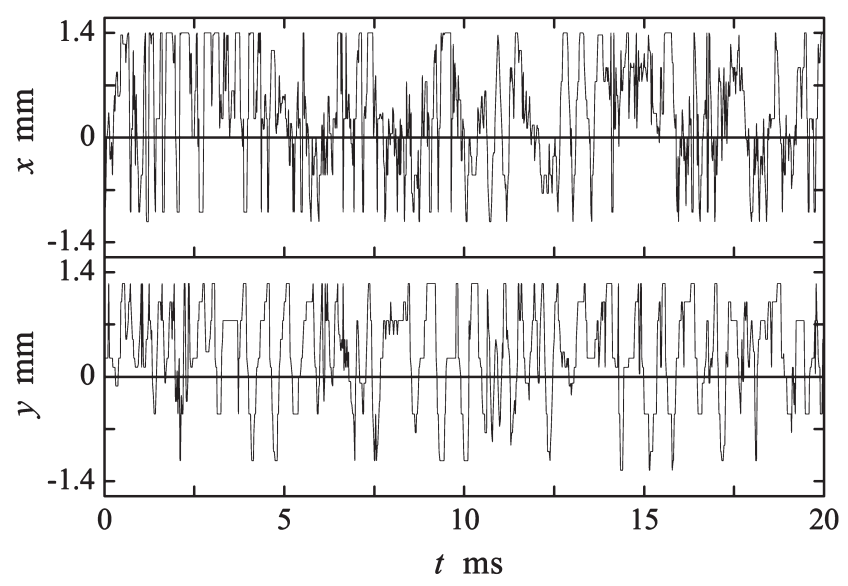

(a) Yarn positions in the $x$ and $y$ directions as a function of time $t$

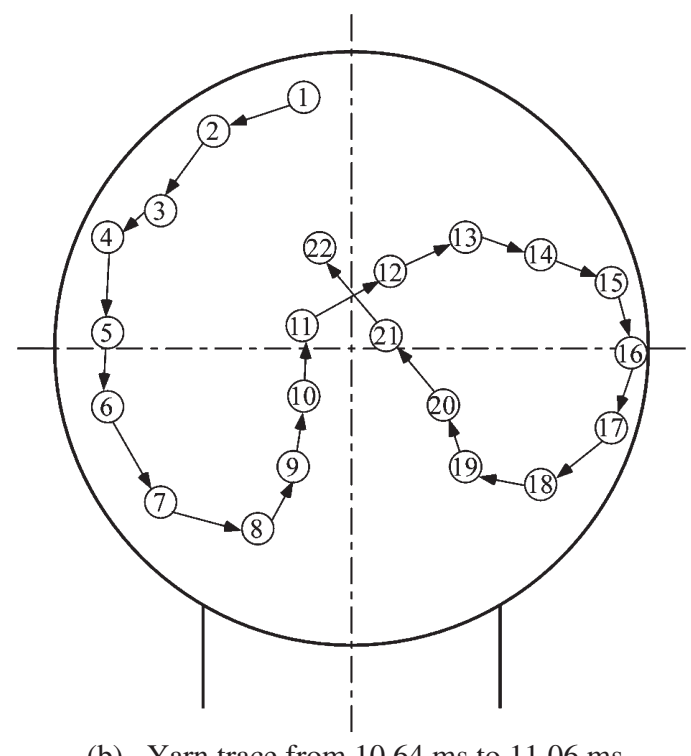

(b) Yarn trace from $10.64 \mathrm{~ms}$ to $11.06 \mathrm{~ms}$

Fig. 8 Yarn motion and trace in the interlacer $\mathrm{C} 1$ at the supplied air pressure of $0.3 \mathrm{MPa}$, the yarn speed of $200 \mathrm{~m} / \mathrm{min}$ and the feed ratio of $2 \%$.

smoothly than in E2. As a result, $S$ in the interlaced yarn produced by the interlacer $\mathrm{C} 1$ becomes small and tangling parts are easy to disentangle. From Figs. 7(b) and 8(b), both the yarn traces are a modified form of a number 8 , which is the same as the result in the previous study [13].

\section{2 Yarn motion in the yarn ducts with a cornered shape}

The interlacers S1, T1 and T2 are classed as the interlacer with a cornered yarn duct. From Fig. 4, among these three interlacers, T2 can produce an interlaced yarn with the largest $N$ and T1 can produce the interlaced yarn with the highest $S$. The performance of $S 1$ is the worst. To compare yarn motions in the interlacers $\mathrm{S} 1, \mathrm{~T} 1$ and $\mathrm{T} 2$, observations were carried out at $p=0.3 \mathrm{MPa}, v=200 \mathrm{~m} / \mathrm{min}$ and $F=2 \%$. 


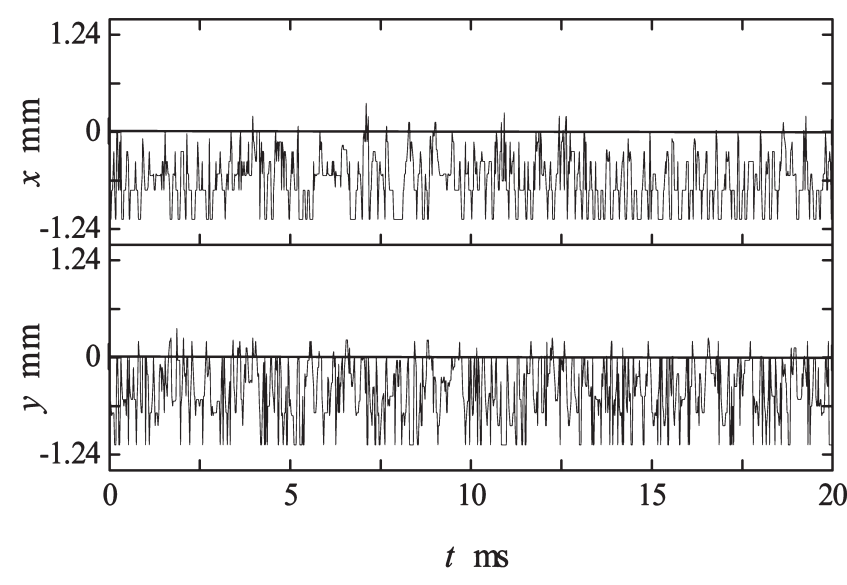

(a) Yarn positions in the $x$ and $y$ directions as a function of time $t$

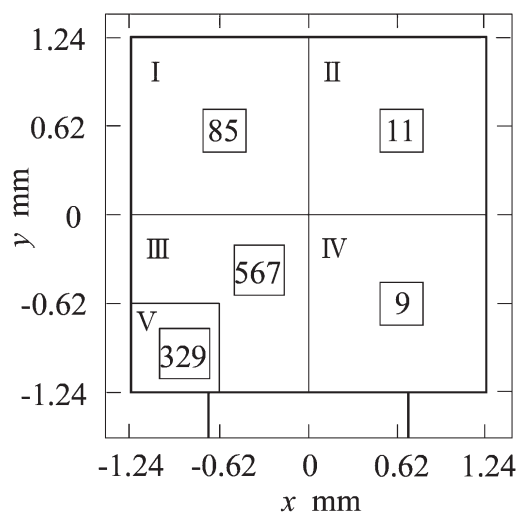

(b) Amount of yarn positions appearing within the five regions for $20 \mathrm{~ms}$

Fig. 9 Yarn motion and position in the interlacer S1 at the supplied air pressure of $0.3 \mathrm{MPa}$, the yarn speed of $200 \mathrm{~m} / \mathrm{min}$ and the feed ratio of $2 \%$.

Figures 9(a) and (b) show the yarn motion and the amount of yarn positions in five regions, $\mathrm{I}: x \leq 0 \mathrm{~mm}$ and $y>0 \mathrm{~mm}$, II : $x>0 \mathrm{~mm}$ and $y>0 \mathrm{~mm}$, III : $x>0 \mathrm{~mm}$ and $y \leq 0 \mathrm{~mm}$, IV : $x \leq 0 \mathrm{~mm}$ and $y \leq 0 \mathrm{~mm}$ except in $x \leq-0.62 \mathrm{~mm}$ and $y \leq-0.62 \mathrm{~mm}$, and $\mathrm{V}: x \leq-0.62 \mathrm{~mm}$ and $y \leq-0.62 \mathrm{~mm}$ for $20 \mathrm{~ms}$ in the interlacer S1. From Fig. 9, yarn mainly stays in the lower corner region of the yarn duct and cannot run across the air jet frequently. In addition, the velocity of the compressed air is smaller in the lower corner regions, which makes yarn opening difficult.

Figure 10 shows the yarn motion in the interlacer T1. Yarn positions change a little in the $x$ direction and yarn stays in the upper half region. Thus the space for yarn opening and tangling is limited, which is not good for yarn interlacing. Compared with S1, yarn in $\mathrm{T} 1$ can run across the air jet frequently. As a result, the performance of $\mathrm{T} 1$ is better than that of $\mathrm{S} 1$.

Figures 11(a) and (b) show the yarn motion and trace from $15.72 \mathrm{~ms}$ to $16.06 \mathrm{~ms}$ in the interlacer $\mathrm{T} 2$, respectively.

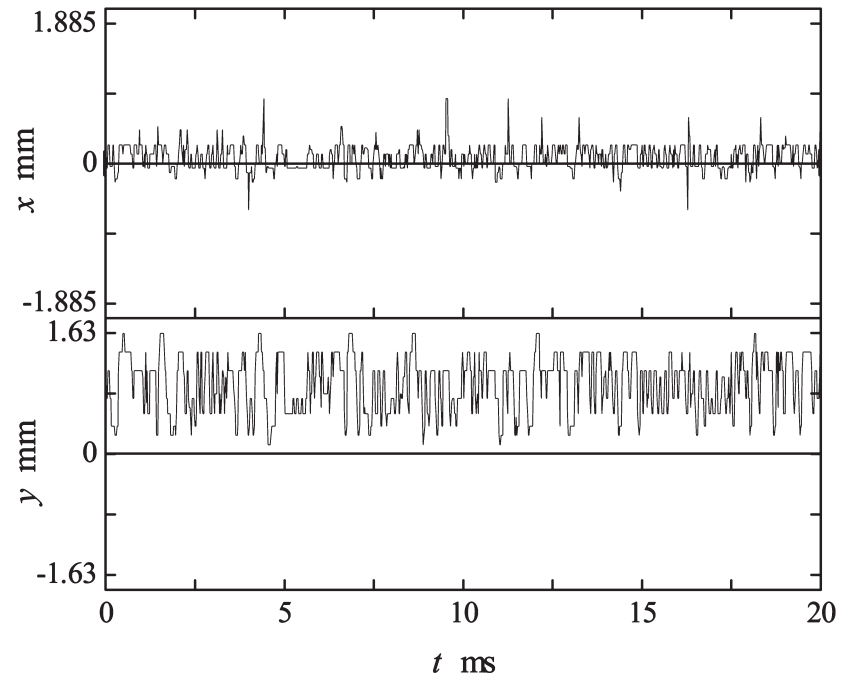

Fig. 10 Yarn motion in the interlacer T1 at the supplied air pressure of $0.3 \mathrm{MPa}$, the yarn speed of $200 \mathrm{~m} / \mathrm{min}$ and the feed ratio of $2 \%$.

Figure 11 (c) shows the amount of yarn positions in four regions, I : $x \leq-0.943 \mathrm{~mm}$, II : $-0.943 \mathrm{~mm}<x<0.943 \mathrm{~mm}$ and $y>0 \mathrm{~mm}$, III $: y \leq 0 \mathrm{~mm}$, IV $: x \geq 0.943 \mathrm{~mm}$. The amount of yarn positions is $31 \%$ in the two corner regions $|x| \geq 0.943 \mathrm{~mm}$. The amount of $31 \%$ is nearly equal to the area ratio $33 \%$ of the two corner regions to the upper half region. Yarn stays in the upper half region, but moves between the two corners and runs across the air jet frequently. Thus the interlacer $\mathrm{T} 2$ can produce an interlaced yarn with larger $N$ than the interlacer T1. On the other hand, the interlacer $\mathrm{T} 1$ can produce an interlaced yarn with higher $S$ than T2 because a yarn is subjected to the direct action of the compressed air in a small space for a long time.

\section{3 Yarn motion in the interlacers E2 and T2}

Among these seven interlacers, as shown in Fig. 4, the interlacers E2 and T2 have better performance than other interlacers because they can produce the interlaced yarn with larger $N$ and appropriate $S$. Yarn in both E2 and T2 moves in a wide space and runs across the air jet frequently. This behavior is good for interlacing.

The interlacer E2 can produce an interlaced yarn with larger $N$ and lower $S$ than T2 from Fig. 4 and Table 1. As shown in Fig. 11(b), a yarn is easy to stay at the top of the yarn duct of T2 and cannot move freely and continuously. Since a yarn in T2 stays for a relatively long time at the top of the yarn duct, the yarn gets much more impingement from the compressed air than that in E2. Thus T2 can produce an interlaced yarn with higher $S$ than E2.

Figures 12(a) and (b) show the yarn motion in the interlacer E2 at $v=200 \mathrm{~m} / \mathrm{min}, F=2 \%, p=0.1 \mathrm{MPa}$ and $p=0.5 \mathrm{MPa}$, respectively, in order to discuss an effect of $p$. 


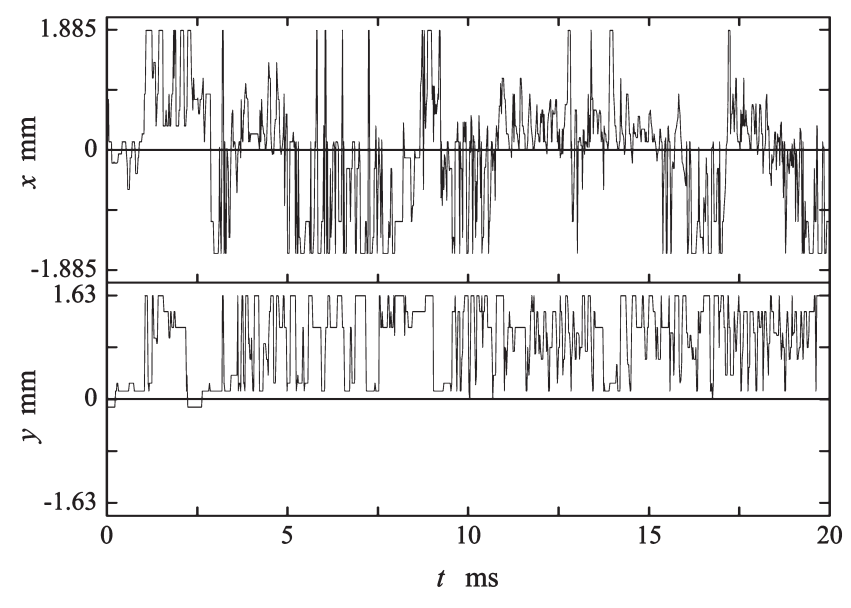

(a) Yarn positions in the $x$ and $y$ directions as a function of time $t$

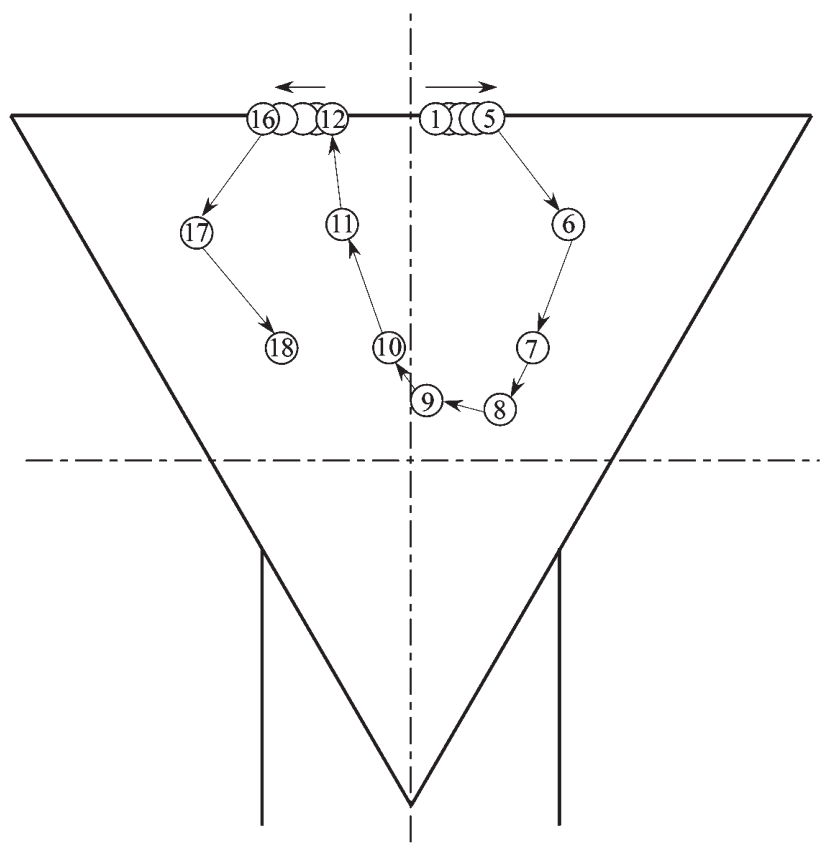

(b) Yarn trace from $15.72 \mathrm{~ms}$ to $16.06 \mathrm{~ms}$

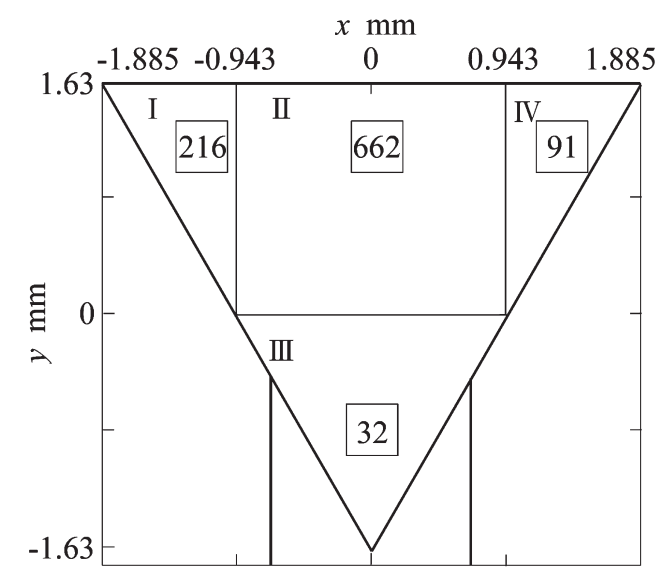

(c) Amount of yarn positions appearing within the four regions for $20 \mathrm{~ms}$

Fig. 11 Yarn motion and trace in the interlacer T2 at the supplied air pressure of $0.3 \mathrm{MPa}$, the yarn speed of $200 \mathrm{~m} / \mathrm{min}$ and the feed ratio of $2 \%$.

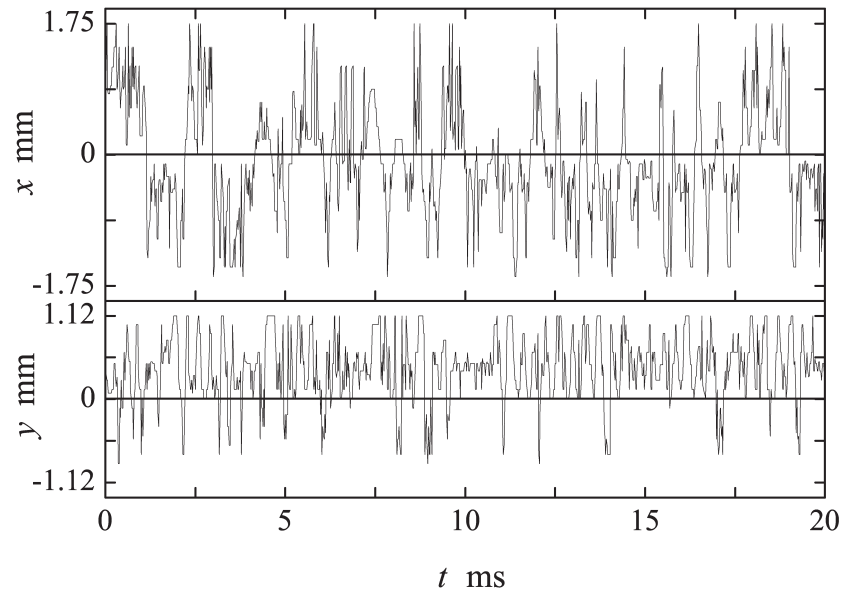

(a) At supplied air pressure of $0.1 \mathrm{MPa}$

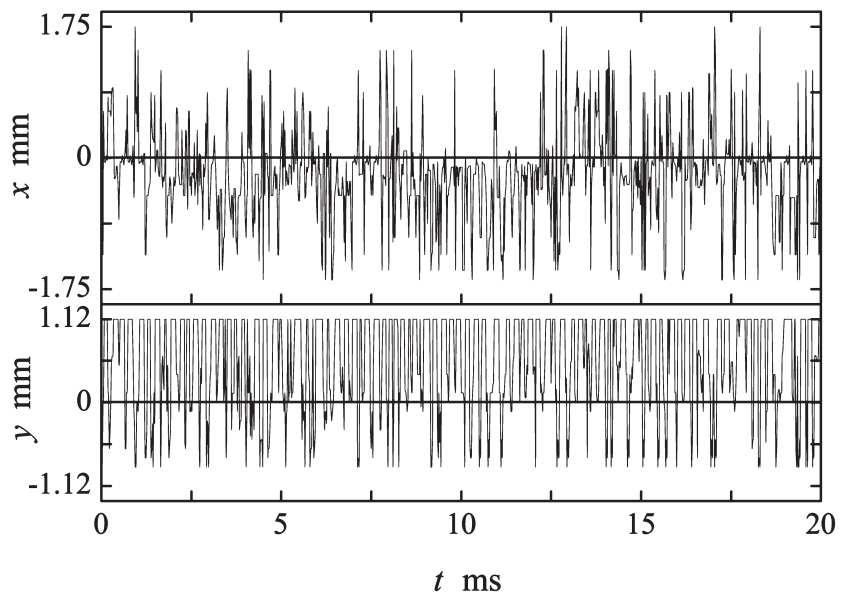

(b) At supplied air pressure of $0.5 \mathrm{MPa}$

Fig. 12 Yarn motion in the interlacer E2 at the yarn speed of $200 \mathrm{~m} / \mathrm{min}$ and the feed ratio of $2 \%$.

At lower supplied air pressure as shown in Fig. 12(a), yarn cannot run across the air jet. At higher supplied air pressure as shown in Fig. 12(b), yarn is easy to be blown to one side of the yarn duct and stays at the top of the yarn duct for a long time. Whereas, when $p=0.3 \mathrm{MPa}$, yarn seldom stays at the top of the yarn duct. Hence, $N$ at $p=0.3 \mathrm{MPa}$ is larger than at both lower and higher $p$.

Figures 13(a) and (b) show the yarn motion in the interlacer T2 at $v=200 \mathrm{~m} / \mathrm{min}, F=2 \%, p=0.1 \mathrm{MPa}$ and $p=0.5 \mathrm{MPa}$, respectively. From Figs. 11(a) and 13, the higher $p$ is, the stronger is the action of airflow and the longer is the time when yarn stays at the top of the yarn duct. Moreover, the every time that yarn stays at the top of the yarn duct of the interlacer T2 is longer than that of the interlacer E2. As a result, the interlacer T2 can produce an interlaced yarn with smaller $N$ than the interlacer E2.

Figures 14 and 15 show the yarn motion in the interlacers E2 and T2 at $p=0.3 \mathrm{MPa}, v=600 \mathrm{~m} / \mathrm{min}$ and $F=2 \%$, 


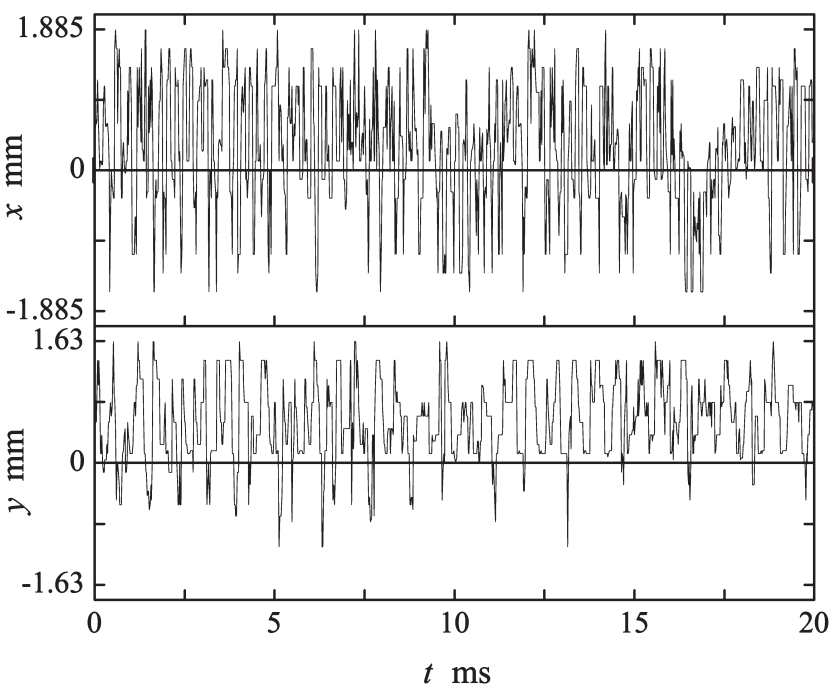

(a) At supplied air pressure of $0.1 \mathrm{MPa}$

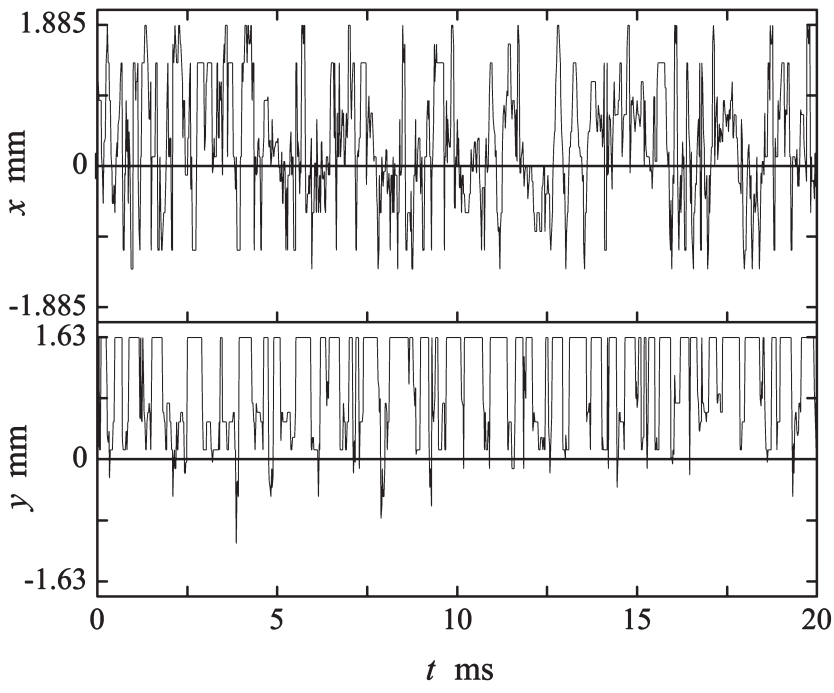

(b) At supplied air pressure of $0.5 \mathrm{MPa}$

Fig. 13 Yarn motion in the interlacer T2 at the yarn speed of $200 \mathrm{~m} / \mathrm{min}$ and the feed ratio of $2 \%$.

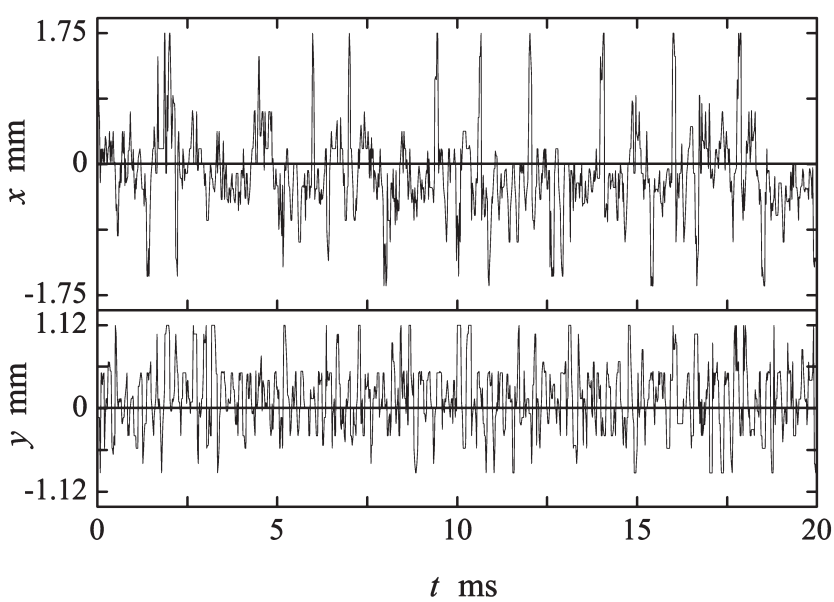

Fig. 14 Yarn motion in the interlacer E2 at the supplied air pressure of $0.3 \mathrm{MPa}$, the yarn speed of $600 \mathrm{~m} / \mathrm{min}$ and the feed ratio of $2 \%$.

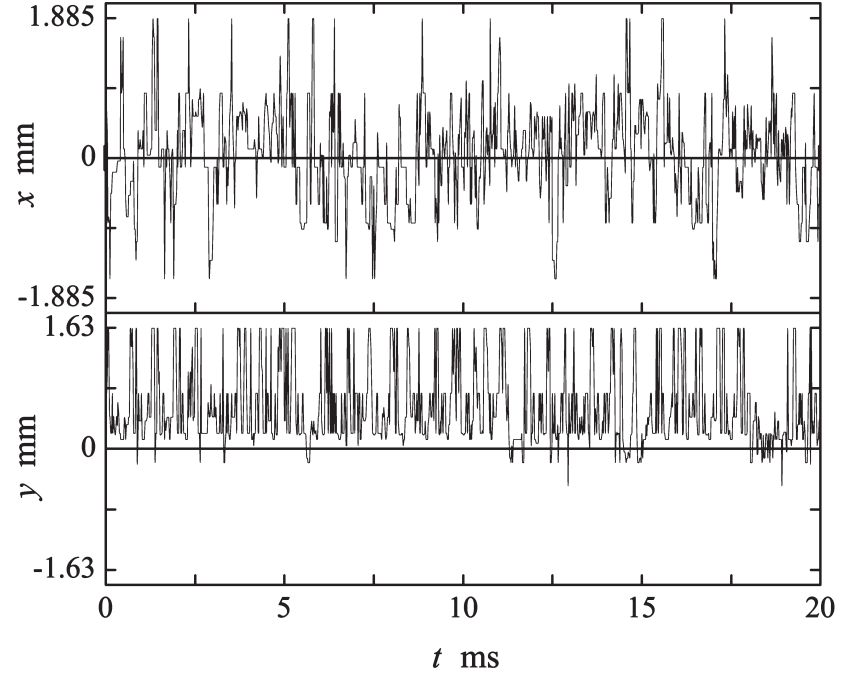

Fig. 15 Yarn motion in the interlacer $\mathrm{T} 2$ at the supplied air pressure of $0.3 \mathrm{MPa}$, the yarn speed of $600 \mathrm{~m} / \mathrm{min}$ and the feed ratio of $2 \%$.

respectively. Comparing Figs. 14 and 15 with Figs. 7(a) and 11(a), respectively, the yarn speed does not influence the yarn motion in the yarn duct essentially. From the previous studies $[4,10]$, higher yarn speed results in small $N$. This is because the period for a yarn to be subjected to the compressed air becomes short [13].

\section{Conclusions}

In order to find the relations between the performance of interlacer and its cross-sectional shapes of yarn duct, yarn motion at the cross-section of yarn duct near the air jet nozzle in the seven interlacers were observed with 16 sets of optical fibers and photo-sensors. Results obtained are as follows:

(1) Yarn motion across the air jet is essential to produce larger $N$ in an interlaced yarn. This is because the interlacers $\mathrm{S} 1$ and $\mathrm{T} 1$ show poor performance.

(2) The interlacers with the round yarn duct, such as E1 and $\mathrm{E} 2$, can make yarn run smoothly in the yarn duct and have better production performance.

(3) The interlacers with the cornered yarn duct, such as T2 and $\mathrm{T} 1$, make yarn stay in a region of a strong airflow for a long time and have the ability to produce higher $S$.

(4) Yarn cannot run across the air jet frequently at lower supplied air pressure. Whereas, the higher supplied air pressure makes yarn stay at the top of the yarn duct for a long time. On these experimental conditions, it is difficult to produce an interlaced yarn with large $N$.

(5) The yarn speed has little effect on the yarn motion.

In the future, we will simulate flow patterns of the compressed air in the yarn duct of these seven interlacers. 


\section{Acknowledgement}

The authors would like to express their thanks to Mr. Shintarou Koizumi, President of Matsubun Textile Co., LTD for kindly supplying the test yarn, Dr. Atsuji Masuda, Researcher of Industrial Technology Center of Fukui Prefecture for kindly supplying the optical fiber, and Mr. Masaharu Tsuji, Engineer of Technical Support Division, Faculty of Engineering, University of Fukui for helping to make the device for measuring yarn motion.

\section{References}

[1] Miao M (1995) Textile Res. J, 65, 433-440

[2] Demir A (1990) Textile Asia, 21, 114-122

[3] Ridarusutoromu R, Wato RJ, Bantingu Jr WW, Neruson TR (1961) Japanese Official Gazette of Patents, Showa 36-12230

[4] Iemoto Y, Chono S, Sawazaki K (1989) J Text Mach Soc
Japan, 35(1), 1-5

[5] Iemoto Y, Chono S, Sawazaki K (1989) J Text Mach Soc Japan, 35(3), 1-5

[6] Denton MJ (1989) Textile Asia, 20, 46-48

[7] Demir A., Acar M (1990) Textile Asia, 21, 77-93

[8] Iemoto Y, Chono S (1997) J Text Mach Soc Japan, 43, 38-46

[9] Iemoto Y, Chono S, Terachi T (1999) J Text Mach Soc Japan, 45, 36-43

[10] Qiu H, Iemoto Y, Tanoue S (2007) J Text Eng, 53, 1-8

[11] Iemoto Y, Chono S, Tanaka T (1998) J Text Mach Soc Japan, 44, 57-63

[12] Iemoto Y, Chono S, Tanaka T (1999) J Text Mach Soc Japan, $45,44-50$

[13] Iemoto Y, Chono S, Kasamatsu K, Lou W (1999) J Text Mach Soc Japan, 45, 71-77

[14] Ge M, Iemoto Y, Tanoue S (1999) J Text Mach Soc Japan, 52, T51-T56 